\title{
A NOTE ON SEPARATING FUNCTION SETS
}

\author{
RAUSHAN BUZYAKOVA AND OLEG OKUNEV
}

\begin{abstract}
We study separating function sets. We find some necessary and sufficient conditions for $C_{p}(X)$ or $C_{p}^{2}(X)$ to have a point-separating subspace that is a metric space with certain nice properties. One of the corollaries to our discussion is that for a zero-dimensional $X, C_{p}(X)$ has a discrete pointseparating space if and only if $C_{p}^{2}(X)$ does.
\end{abstract}

\section{INTRODUCTION}

To start our discussion, recall, that $F \subset C_{p}^{n}(X)$ is point-separating if for any distinct $x, y \in X$ there exists $\left\langle f_{1}, \ldots, f_{n}\right\rangle \in F$ such that $f_{i}(x) \neq f_{i}(y)$ for some $i \leq n$. In this paper we are concerned with the following general problem.

Problem. Let $P$ be a nice property. Describe " $C_{p}(X)$ (or $C_{p}^{m}(X)$ ) having a point-separating subspace with $P$ " in terms of the topology of $X, X^{n}$, or $X^{\omega}$.

In this study, $P$ is the property of being a discrete space, a countable union of discrete subspaces, a metric compactum, or a discrete group. We obtain two characterizations of spaces $X$ for which $C_{p}^{2}(X)$ has a discrete point-separation subspace (Theorems 2.9] and 2.10, and 2.17). One of the characterizations is consistent and may have a chance for a ZFC proof. We also characterize zerodmensional $Z$ with point-separating discrete subspaces in $C_{p}(X)$ (Theorems 2.13 and 2.14, and 2.18). Questions of similar nature are quite popular among topologists interested in $C_{p}$-theory and have been considered in many papers.

In notation and terminology we follow [2]. All spaces under consideration are assumed Tychonoff and infinite. By $s(X)$ we denote the supremum of cardinalities of discrete subspaces of $X$. By $i w(X)$ we denote the smallest weight of a Tychonoff subtopology of $X$. When we say that $D$ is a discrete subspace of $X, D$ need not be closed in $X$. By $\sigma_{X}\left(x^{*}\right)$ we denote the subspace of $X^{\omega}$ that consists of all points that differ from $x^{*}$ by finitely many coordinates. Since $\sigma_{X}(x)$ and $\sigma_{X}(y)$ are obviously homeomorphic we may simply write $\sigma_{X}$ and, as usual, refer to it as $\sigma$-product of $X^{\omega}$. A standard neighborhood of $f$ in $C_{p}(X)$

1991 Mathematics Subject Classification. 54C35, 54E45, 54A25.

Key words and phrases. $C_{p}(X)$, discrete space, point-separating set, spread, $i$-weight, $\sigma$ product. 
is in form $U\left(x_{1}, \ldots, x_{n} ; B_{1}, \ldots, B_{n}\right)=\left\{g \in C_{p}(X): g\left(x_{i}\right) \in B_{i}\right\}$, where $B_{i}$ is open interval with rational endpoints for each $i$. Zero-dimensionality is understood in the sense of dim.

\section{Discrete Point-Separating Subspaces}

Our first goal is to find a characterization of those infinite $X$ for which $C_{p}(X)$ or $C_{p}^{2}(X)$ has a point-separating discrete subspace. We achieve the goal within a wide class of spaces. We start with a few helping lemmas.

The following Lemma is almost identical to Proposition II.5.5 in [1] but due to cofinality restrictions we have to prove it using a similar argument.

Lemma 2.1. (version of [1, II.5.5]) Assume that $C_{p}^{2}(X)$ has a discrete subspace of size $\tau$. Then the following hold:

(1) If $c f(\tau)>\omega$, then $s\left(X^{n}\right) \geq \tau$ for some $n \in \omega$.

(2) If $c f(\tau)=\omega$, then $s\left(\sigma_{X}\right) \geq \tau$.

Proof. Since part (2) is an obvious consequence of part (1), we will prove part (1) only. Let $D \subset C_{p}^{2}(X)$ be a $\tau$-sized discrete subspace. For each $\langle f, g\rangle \in D$ fix standard neighborhoods $U_{f}=U_{f}\left(x_{1}^{f}, \ldots, x_{n_{f}}^{f}, I_{1}^{f}, \ldots, I_{n_{f}}^{f}\right)$ and $V_{g}=V_{g}\left(y_{1}^{g}, \ldots, y_{m_{g}}^{g}, J_{1}^{g}, \ldots, J_{m_{g}}^{g}\right)$ such that $U_{f} \times V_{g}$ contains $\langle f, g\rangle$ and misses $D \backslash\{\langle f, g\rangle\}$. Since $c f(\tau)>\omega$ we can find $n^{*}, m^{*} \in \omega,\left\langle I_{i}: i \leq n^{*}\right\rangle$, $\left\langle J_{i}: i \leq m^{*}\right\rangle$, and a $\tau$-sized $D^{\prime} \subset D$ such that $n_{f}=n^{*}, m_{g}=m^{*}$, $\left\langle I_{i}^{f}: i \leq n^{*}\right\rangle=\left\langle I_{i}: i \leq n^{*}\right\rangle$, and $\left\langle J_{i}^{g}: i \leq m^{*}\right\rangle=\left\langle J_{i}: i \leq m^{*}\right\rangle$ for each $\langle f, g\rangle \in D^{\prime}$. We can now conclude that for any distinct $\langle f, g\rangle,\left\langle f^{\prime}, g^{\prime}\right\rangle \in D^{\prime}$, either $\left\langle x_{i}^{f}: i \leq n^{*}\right\rangle \neq\left\langle x_{i}^{f^{\prime}}: i \leq n^{*}\right\rangle$ or $\left\langle y_{i}^{g}: i \leq m^{*}\right\rangle \neq\left\langle y_{i}^{g^{\prime}}: i \leq m^{*}\right\rangle$. Therefore, the set $S=\left\{\left\langle x_{i}^{f}, \ldots, x_{n^{*}}^{f}, y_{i}^{g}, \ldots, y_{m^{*}}^{g}\right\rangle:\langle f, g\rangle \in D^{\prime}\right\}$ is $\tau$-sized. To show that $S$ is a discrete subspace of $X^{n^{*}+m^{*}}$, for each $\langle f, g\rangle \in D^{\prime}$, put $U_{f}=f^{-1}\left(I_{1}\right) \times \ldots \times f^{-1}\left(I_{n^{*}}\right)$ and $V_{g}=g^{-1}\left(J_{1}\right) \times \ldots \times g^{-1}\left(J_{m^{*}}\right)$. Clearly $U_{f} \times V_{g}$ is a neighborhood of $\left\langle x_{i}^{f}, \ldots, x_{n^{*}}^{f}, y_{i}^{g}, \ldots, y_{m^{*}}^{g}\right\rangle$ in $X^{n^{*}+m^{*}}$. Next, fix $\left\langle f^{\prime}, g^{\prime}\right\rangle \in D^{\prime} \backslash\{\langle f, g\rangle\}$. By the choice of our neighborhoods, we may assume that $f \notin U_{f^{\prime}}$. Therefore, there exists $i \leq n^{*}$ such that $f\left(x_{i}^{f^{\prime}}\right) \notin I_{i}$. Therefore, $x_{i}^{f^{\prime}} \notin f^{-1}\left(I_{i}\right)$, which implies that $\left\langle x_{i}^{f^{\prime}}, \ldots, x_{n^{*}}^{f^{\prime}}, y_{i}^{g^{\prime}}, \ldots, y_{m^{*}}^{g^{\prime}}\right\rangle \notin U_{f} \times V_{g}$.

Note that if $C_{p}(X)$ or $C_{p}^{2}(X)$ has a discrete point-separating subspace of an infinite size $\tau$, then $\tau \geq i w(X)$. If in addition $c f(\tau)>\omega$, then, by Lemma 2.1, $s\left(X^{n}\right) \geq \tau \geq i w(X)$ for some $n$. Thus, the following statement holds. 
Theorem 2.2. Assume that $C_{p}(X)$ or $C_{p}^{2}(X)$ has a discrete point-separating subspace of size $\tau$ with $c f(\tau)>\omega$. Then $s\left(X^{n}\right) \geq \tau \geq i w(X)$ for some natural number $n$.

We are now ready to formulate and prove two necessary conditions for $C_{p}(X)$ and $C_{p}^{2}(X)$ to have a point-separating discrete subspace.

Theorem 2.3. If $C_{p}^{2}(X)$ has a discrete point-separating subspace, then $s\left(\sigma_{X}\right) \geq$ $i w(X)$.

Proof. Put $\tau=i w(X)$. If $\tau$ is countable, then $X$ has a countable network. Since $X$ is infinite, it contains an infinite countable subspace. Hence, $s\left(\sigma_{X}\right) \geq i w(X)$.

We now assume that $\tau$ is uncountable. By Theorem 2.2 we may assume that $c f(\tau)=\omega$. Fix a strictly increasing sequence of cardinals $\tau_{n}$ of uncountable cofinalities so that $\tau=\sum_{n} \tau_{n}$. Since any point-separating subset of $C_{p}^{2}(X)$ must have size at least $\tau$, there exists a discrete subset of cardinality $\tau_{n}$ in $C_{p}^{2}(X)$ for each $n$. By Lemma 2.1, there exists a discrete subset $D_{n}$ in some finite power of $X$ for each $n$. Therefore, $s\left(\sigma_{X}\right) \geq \tau$.

In all future arguments, the cases when a discrete point-separating subspace is finite can be handled as in Theorem 2.3 and will therefore not be considered. For our next observation we need Zenor's theorem [7] stating that if $s(X \times Y) \leq$ $\tau \geq \omega$ then either $h l(X) \leq \tau$ or $h d(Y) \leq \tau$.

Theorem 2.4. Assume Generalized Continuum Hypothesis. If $C_{p}^{2}(X)$ has a discrete point-separating subspace, then $s\left(X^{n}\right) \geq i w(X)$ for some $n \in \omega$.

Proof. Put $\tau=i w(X)$. By Theorem 2.2 we may assume that $\tau$ is an infinite cardinal of countable cofinality. Assume the contrary. Then $s\left(X^{4}\right)=\lambda<\tau$. By Zenor's theorem, $h l\left(X^{2}\right) \leq \lambda$ or $d\left(X^{2}\right) \leq \lambda$. If the former is the case, then the off-diagonal part of $X^{2}$ can be covered by $\lambda$-many functionally closed boxes, which implies that $i w(X)<\tau$. If $d\left(X^{2}\right) \leq \lambda$, then by Generalized Continuum Hypothesis, $w\left(X^{2}\right)$ is at most $2^{\lambda}<\tau$. Since both cases lead to contradictions, the statement is proved.

The assumptions in Theorem 2.4 prompts the following questions.

Question 2.5. Does Theorem 2.4 hold in ZFC? 
Note that if one can construct a space $X$ such that $s\left(X^{n}\right)=\omega_{n}$ for all natural numbers $n$ and $i w(X)=\omega_{\omega}$, then the answer to Question 2.5 is a "no".

At this point one may wonder if our study is justified. In other words, are we studying a non-empty class? Let $X$ be an non-metrizable compact space such that $X^{n}$ is hereditary separable for each $n$. Such a space exists. A consistent example of such a space is Ivanov's modification [5] of Fedorchuk's example [3]. Since $X^{n}$ is hereditarily separable, by Lemma 2.1. no discrete subspace of $C_{p}^{2}(X)$ or $C_{p}(X)$ is uncountable. Since $X$ is not submetrizable, we conclude that no countable subspace of $C_{p}^{2}(X)$ or $C_{p}(X)$ is point-separating. Let us summarize this observation as follows.

Example 2.6. There exists a consistent example of a compactum $X$ such that neither $C_{p}(X)$ nor $C_{p}^{2}(X)$ has a discrete separating subspace.

The authors believe that in some models of ZFC, no such example may exist, meaning that any space may have a discrete in itself point-separating function set.

Question 2.7. Is there a ZFC example of a space $X$ such that no discrete subspace of $C_{p}(X)\left(C_{p}^{n}(X)\right)$ is point-separating?

We will next reverse the statement of Theorem 2.2, which will bring us to the promised characterizations.

Theorem 2.8. If $X^{n}$ has a discrete subspace of size iw $(X)$ for some natural number $n$, then $C_{p}^{2}(X)$ has a point-separating discrete subspace.

Proof. Let $n$ be the smallest that satisfies the hypothesis of the lemma and put $\tau=i w(X)$. By the choice of $n$ there exists a $\tau$-sized discrete subspace $D$ of $X^{n}$ with the following property:

$$
\text { Property: }|\{x(i): i \leq n\}|=n \text { for each } x \in D \text {. }
$$

Let $\mathcal{T}$ be a Tychonoff subtopology of the topology of $X$ of weight $\tau$. Fix a $\tau$-sized network $\mathcal{N}$ for $\langle X, \mathcal{T}\rangle$ that consists of functionally closed subsets. Let $\mathcal{P}$ be the set of all pairs $\langle A, B\rangle$ of disjoint elements of $\mathcal{N}$. Enumerate $\mathcal{P}$ and $D$ as $\left\{\left\langle A_{\alpha}, B_{\alpha}\right\rangle: \alpha<\tau\right\}$ and $\left\{d_{\alpha}: \alpha<\tau\right\}$. Since $D$ is a discrete subspace, for each $\alpha<\tau$ we can fix a functionally closed set $B_{1}^{\alpha} \times \ldots \times B_{n}^{\alpha}$ that contains $d_{\alpha}$ in its interior and misses $D \backslash\left\{d_{\alpha}\right\}$. By Property, we may assume that $B_{i}^{\alpha} \cap B_{j}^{\alpha}=\emptyset$ for distinct $i$ and $j$.

We will next construct our desired subspace $\left\{\left\langle f_{\alpha}, g_{\alpha}\right\rangle: \alpha<\tau\right\}$ of $C_{p}(X)$. 
Definition of $f_{\alpha}$, where $\alpha<\tau$ : Let $S_{\alpha}$ be a functionally closed subset of $X$ such that $X \backslash S_{\alpha}$ can be written as a union of $L_{\alpha}$ and $R_{\alpha}$ so that the following hold.

(1) $c l_{X}\left(L_{\alpha}\right) \cap c l_{X}\left(R_{\alpha}\right) \subset S_{\alpha}$

(2) $A_{\alpha} \subset L_{\alpha}$ and $B_{\alpha} \subset R_{\alpha}$;

(3) $d_{\alpha}(i) \in L_{\alpha}$ if $d_{\alpha}(i) \notin B_{\alpha}$, and $d_{\alpha}(i) \in R_{\alpha}$ if $d_{\alpha}(i) \in B_{\alpha}$.

Such an $S_{\alpha}$ exists since $A_{\alpha}$ and $B_{\alpha}$ are functionally separable sets and the coordinate set of $d_{\alpha}$ is finite. Let $f_{\alpha, l}: L_{\alpha} \cup S_{\alpha} \rightarrow[-1,0]$ be any continuous function that has the following properties:

L1: $f_{\alpha, l}^{-1}(\{0\})=S_{\alpha}$;

L2: $\left(\left\{d_{\alpha}(i): i \leq n\right\} \cap L_{\alpha}\right) \subset f_{\alpha, l}^{-1}([-1,-1 / 3)) \subset \bigcup\left\{B_{i}^{\alpha}: d_{\alpha}(i) \in L_{\alpha}\right\}$. Such a function exists because the coordinate set of $d_{\alpha}$ is finite and $S_{\alpha}$ is functionally closed and misses the coordinate set of $d_{\alpha}$. Let $f_{\alpha, r}$ : $R_{\alpha} \cup S_{\alpha} \rightarrow[0,1]$ be any continuous function that has the following properties:

$\mathrm{R} 1: f_{\alpha, r}^{-1}(\{0\})=S_{\alpha} ;$

$\mathrm{R} 2:\left(\left\{d_{\alpha}(i): i \leq n\right\} \cap R_{\alpha}\right) \subset f_{\alpha, r}^{-1}((1 / 3,1]) \subset \bigcup\left\{B_{i}^{\alpha}: d_{\alpha}(i) \in R_{\alpha}\right\}$.

Put $f_{\alpha}=f_{\alpha, l} \cup f_{\alpha, r}$. By L1 and R1, $f_{\alpha}$ is a continuous function from $X$ to $\mathbb{R}$.

Definition of $g_{\alpha}$, where $\alpha<\tau$ : Let $g_{\alpha}$ be any continuous function that maps $B_{i}^{\alpha}$ to $(i-1 / 3, i+1 / 3)$. This can be done since $B_{i}^{\alpha}$ 's form a disjoint finite collection of functionally closed sets.

It remains to show that $F=\left\{\left\langle f_{\alpha}, g_{\alpha}\right\rangle: \alpha<\tau\right\}$ is a point-separating discrete subspace. To show that $F$ is point-separating, fix distinct $a, b$ in $X$. Since $\mathcal{N}$ is a network, there exist disjoint $A, B \in \mathcal{N}$ that contain $a$ and $b$, respectively. Then $\langle A, B\rangle=\left\langle A_{\alpha}, B_{\alpha}\right\rangle \in \mathcal{P}$. By the definition of $f_{\alpha}, f_{\alpha}(a)=f_{\alpha, l}(a)<0$ and $f_{\alpha}(b)=f_{\alpha, r}(b)>0$.

To show that $F$ is discrete in itself, fix $\alpha$. Put

$$
\begin{gathered}
U_{\alpha}=\left\{f: f\left(d_{\alpha}(i)\right)<-1 / 3 \text { if } d_{\alpha}(i) \in L_{\alpha}, f\left(d_{\alpha}(i)\right)>1 / 3 \text { if } d_{\alpha}(i) \in R_{\alpha}\right\} \\
V_{\alpha}=\left\{g: g\left(d_{\alpha}(i)\right) \in(i-1 / 3, i+1 / 3)\right\}
\end{gathered}
$$

Clearly, $U_{\alpha} \times V_{\alpha}$ is a neighborhood of $\left\langle f_{\alpha}, g_{\alpha}\right\rangle$. To show that this neighborhood misses the rest of $F$, fix $\beta \neq \alpha$. There exists $i \leq n$ such that $d_{\alpha}(i) \notin B_{i}^{\beta}$. We have two possible cases.

Case 1: This case's assumption is that $d_{\alpha}(i) \notin \bigcup_{j \leq n} B_{j}^{\beta}$. By L2 and R2 of the definition of $f_{\beta}$, we have $f_{\beta}\left(\left(d_{\alpha}^{i}\right)\right) \in(-1 / 3,1 / 3)$. Hence $f_{\beta} \notin U_{\alpha}$. Therefore, $\left\langle f_{\beta}, g_{\beta}\right\rangle \notin U_{\alpha} \times V_{\alpha}$.

Case 2: Assume Case 1 does not take place. Then there exists $j \leq n$ such that $d_{\alpha}(i) \in B_{j}^{\beta}$. By the choice of $i$, we have $i \neq j$. Therefore, 
$g_{\beta}\left(d_{\alpha}(i)\right) \notin(i-1 / 3, i+1 / 3)$. Hence $g_{\beta} \notin V_{\alpha}$. Therefore, $\left\langle f_{\beta}, g_{\beta}\right\rangle \notin$ $U_{\alpha} \times V_{\alpha}$.

Statements 2.2, 2.8, and 2.4 result in the following criteria.

Theorem 2.9. Let a space $X$ have iw $(X)$ of uncountable cofinality. Then $C_{p}^{2}(X)$ has a point-separating discrete subspace if and only if $s\left(X^{n}\right) \geq i w(X)$ for some $n$.

Theorem 2.10. Assume Generalized Continuum Hypothesis. Then $C_{p}^{2}(X)$ has a point-separating discrete subspace if and only if $s\left(X^{n}\right) \geq i w(X)$ for some $n$.

Note that criteria 2.9 and 2.10 would hold for $C_{p}(X)$ if we could prove Theorem 2.8 for $C_{p}(X)$.

Question 2.11. Assume that $X^{n}$ has a discrete subspace of size iw $(X)$ for some natural number $n$. Is it true that $C_{p}(X)$ has a discrete point-separating set?

Using an argument somewhat similar to that of Theorem 2.8 we will next show that Question 2.11 has an affirmative answer if we assume that $C$ is zerodimensional.

Theorem 2.12. Assume that $X$ is zero-dimensional. If $X^{n}$ has a discrete subspace of size iw $(X)$, then $C_{p}(X)$ has a point-separating discrete subspace.

Proof. Let $n$ be the smallest that satisfies the hypothesis of the lemma and put $\tau=i w(X)$. By the choice of $n$ there exists a $\tau$-sized discrete subspace $D$ of $X^{n}$ with the following property:

Property: $|\{x(i): i \leq n\}|=n$ for each $x \in D$.

Let $\mathcal{T}$ be a Tychonoff subtopology of the topology of $X$ of weight $\tau$. Due to zero-dimensionality of $X$ and the factorization theorem of Mardesic [6], we may assume that $\mathcal{T}$ is zero-dimensional too. Fix a $\tau$-sized network $\mathcal{N}$ for $\langle X, \mathcal{T}\rangle$ that consists of clopen subsets. Let $\mathcal{P}$ be the set of all pairs $\langle A, B\rangle$ of disjoint elements of $\mathcal{N}$. Enumerate $\mathcal{P}$ and $D$ as $\left\{\left\langle A_{\alpha}, B_{\alpha}\right\rangle: \alpha<\tau\right\}$ and $\left\{d_{\alpha}: \alpha<\tau\right\}$. We will next construct our desired subspace in $C_{p}(X)$.

Definition of $f_{\alpha}$, where $\left.\alpha<\tau\right]$ Since $D$ is a discrete subspace, we can fix a clopen box $U_{1}^{\alpha} \times \ldots \times U_{n}^{\alpha}$ that contains $d_{\alpha}$ and misses $D \backslash\left\{d_{\alpha}\right\}$. By Property, we may assume that $U_{i}^{\alpha} \cap U_{j}^{\alpha}=\emptyset$ if $i \neq j$. Since $A_{\alpha}$ and $B_{\alpha}$ are disjoint, 
we may assume that each $U_{i}$ meets at most one of the sets $A_{\alpha}$ and $B_{\alpha}$. Define $f_{\alpha}: X \rightarrow\{0,1,2, \ldots, n+1\}$ by letting $f_{\alpha}\left(U_{i}\right)=\{i\}, f_{\alpha}\left(A_{\alpha} \backslash \bigcup_{i \leq n} U_{i}\right)=\{0\}$, and $f_{\alpha}\left(X \backslash\left(A_{\alpha} \cup U_{1} \cup \ldots \cup U_{n}\right)\right)=\{n+1\}$.

It remains to show that $F=\left\{f_{\alpha}: \alpha<\tau\right\}$ is a point-separating discrete subspace. To show that $F$ is point-separating, fix distinct $a, b$ in $X$. Since $\mathcal{N}$ is a network, there exist disjoint $A, B \in \mathcal{N}$ that contain $a$ and $b$, respectively. Then $\langle A, B\rangle=\left\langle A_{\alpha}, B_{\alpha}\right\rangle \in \mathcal{P}$. Since no $U_{i}^{\alpha}$ meets both $A_{\alpha}$ and $B_{\alpha}$ at the same time, $f_{\alpha}\left(A_{\alpha}\right)$ misses $f_{\alpha}\left(B_{\alpha}\right)$.

To show that $F$ is discrete in itself, fix $f_{\alpha}$ and put $V_{\alpha}=\left\{f: f\left(d_{\alpha}(i)\right) \in\right.$ $(i-1 / 3, i+1 / 3), i \leq n\}$. Next fix any $\beta \neq \alpha$. Then there exists $i \leq n$ such that $d_{\alpha}(i) \notin U_{i}^{\beta}$. Therefore, $f_{\beta}\left(d_{\alpha}(i)\right) \notin(i-1 / 3, i+1 / 3)$. Hence, $f_{\beta} \notin U_{\alpha}$.

Note that Theorems 2.9 and 2.10 are now true for $C_{p}(X)$ provided $X$ is zerodimensional. Let us state the new versions for reference.

Theorem 2.13. Let a zero-dimensional space $X$ have iw $(X)$ of uncountable cofinality. Then $C_{p}(X)$ has a point-separating discrete subspace if and only if $s\left(X^{n}\right) \geq i w(X)$ for some natural number $n$.

Theorem 2.14. Assume Generalized Continuum Hypothesis. Let $X$ be zerodimensional. Then $C_{p}(X)$ has a point-separating discrete subspace if and only if $s\left(X^{n}\right) \geq i w(X)$ for some $n$.

For our final characterization discussion we would like to extract a technical statement from the proof of Theorem 2.8 and prove one helpful proposition.

Lemma 2.15. Assume that a finite power of $X$ has a discrete subspace of size $\lambda$. Let $\left\{\left\langle A_{\alpha}, B_{\alpha}\right\rangle: \alpha<\lambda\right\}$ be a family of pairs of functionally closed disjoint subsets of $X$. Then there exists a discrete subspace $F$ in $C_{p}^{2}(X)$ with the following property:

$\left(^{*}\right)$ If $a \in A_{\alpha}$ and $b \in B_{\alpha}$ for some $\alpha<\lambda$, then $f(a) \neq f(b)$ for some $f \in F$.

Proposition 2.16. Let $C_{p}^{m}(X)$ contain a point-separating subspace which is a countable union of discrete subspaces. Then $C_{p}^{m}(X)$ has a discrete pointseparating subspace. 
Proof. We will prove the statement for $m=2$. Let $D=\cup_{n} D_{n}$ be a pointseparating set of $C_{p}^{2}(X)$, where each $D_{n}$ is a discrete subspace. For each $n$, fix a homeomorphism $h_{n}: \mathbb{R} \rightarrow(n, n+1)$. Put $E_{n}=\left\{\left\langle h_{n} \circ f, h_{n} \circ g\right\rangle:\langle f, g\rangle \in D_{n}\right\}$. Clearly, $E_{n}$ separates $x$ and $y$ if and only if $D_{n}$ does. Also, $E_{n}$ is a discrete subspace of $C_{p}^{2}(X)$. Since all functions in $\left(\cup_{k} E_{k}\right) \backslash E_{n}$ target $\mathbb{R} \backslash(n, n+1)$, we conclude that the closure of $\left(\cup_{k} E_{k}\right) \backslash E_{n}$ misses $E_{n}$. Terefore, $\cup_{n} E_{n}$ is a point-separating discrete subspace of $C_{p}^{2}(X)$.

Theorem 2.17. $C_{p}^{2}(X)$ has a discrete point-separating subspace if and only if $s\left(\sigma_{X}\right) \geq i w(X)$.

Proof. Necessity is done in Theorem 2.3. To prove sufficiency, put $\tau=i w(X)$. Let $\mathcal{N}$ be a $\tau$-sized family of functionally closed subsets of $X$ that is a network for some Tychonoff subtopology of $X$. Let $\mathcal{P}$ consist of all pairs of disjoint elements of $\mathcal{N}$. For each $n$ we can find a discrete subset $D_{n}$ of $\sigma_{X}$ that lives in a copy of some finite power of $X$ so that $\tau=\sum_{n}\left|D_{n}\right|$. Next represent $\mathcal{P}$ as $\bigcup \mathcal{P}_{n}$, where $\left|\mathcal{P}_{n}\right|=\left|D_{n}\right|$. Applying Lemma 2.15 to $\mathcal{P}_{n}$ and $D_{n}$ for each $n$, we find a point-separating subspace in $C_{p}^{2}(X)$ that is a countable union of discrete subspaces. By Proposition [2.16, $C_{p}^{2}(X)$ contains a discrete point-separating subspace.

An argument identical to that of Theorem 2.17 leads to the following statement for $C_{p}(X)$.

Theorem 2.18. Assume that $X$ is zero-dimensional. Then $C_{p}(X)$ has a discrete point-separating subspace if and only if $s\left(\sigma_{X}\right) \geq i w(X)$.

Theorems 2.17 and 2.18 imply the following.

Corollary 2.19. Let $X$ be a zero-dimensional space. Then $C_{p}(X)$ has a pointseparating discrete subspaces if and only if $C_{p}^{2}(X)$ does.

Note that the image of a point-separating family under a homeomorphism need not be point-separating. Indeed, $\left\{i d_{[0,1]}\right\}$ is a point-separating subspace of $C_{p}([0,1])$, However, one can construct an automorphism on $C_{p}([0,1])$ that caries $\left\{i d_{[0,1]}\right\}$ to $\{\overrightarrow{0}\}$ which is not point-separating. In connection with this observation, it would be interesting to know if having a discrete point-separating subspace is preserved by homeomorphisms among function spaces. The answer is affirmative and to show it we will use the fact [1, I,1,6] that if $C_{p}(X)$ and $C_{p}(Y)$ are homeomorphic then $i w(X)=i w(Y)$. 
Theorem 2.20. Let $X$ and $Y$ be t-equivalent. If $C_{p}^{2}(X)$ has a discrete pointseparating subspace, then so does $C_{p}^{2}(Y)$.

Proof. Fix a homeomorphism $\phi: C_{p}^{2}(X) \rightarrow C_{p}^{2}(Y)$ and a discrete pointseparating subspace $D$ of $C_{p}^{2}(X)$.

Assume, first, that $|D|$ is finite. Then $i w(X)=\omega$. Hence $i w(Y)=\omega$. Since $Y$ is infinite, it contains a an infinite countable subspace. By Theorem 2.17, $C_{p}^{2}(Y)$ contains a discrete point-separating subspace.

We now assume that $|D|$ is infinite. Then $|D| \geq i w(X)$. Therefore, $|\phi(D)| \geq$ $i w(Y)$. By Lemma 2.1, $s\left(\sigma_{Y}\right) \geq|\phi(D)| \geq i w(Y)$. By Theorem 2.17, $C_{p}^{2}(Y)$ contains a discrete point-separating subspace.

Repeating the argument of Theorem 2.20, we obtain the following.

Theorem 2.21. Let $X$ and $Y$ be zero-dimensional and t-equivalent. If $C_{p}(X)$ has a discrete point-separating subspace, then so does $C_{p}(Y)$.

While being a discrete subspace is already a nice property, it would be interesting to know when $C_{p}(X)$ or its finite power has a discrete point-separating subspace which is in addition a subgroup. Note that any discrete subgroup is closed. In addition, $C_{p}(X)$ can be covered by countably many shifts of any neighborhood of zero-function. Therefore, any discrete subgroup of $C_{p}(X)$ is countable. These observations lead to the following question.

Question 2.22. Let $X$ be a separable metric space. Is it true that $C_{p}(X)$ has a discrete point-separating subgroup?

It is worth noting that separable metric spaces have many pretty pointseparating subspaces as backed up by the next two statement.

Theorem 2.23. $C_{p}(X)$ has a point-separating subset homeomorphic to $[0,1]$ if and only $X$ admits a continuous injection into $\mathbb{R}^{\omega}$.

Proof. To prove necessity, let $F \subset C_{p}(X)$ a point-separating family homeomorphic to $[0,1]$. Then any dense subset of $F$ is point-separating too. Therefore, $C_{p}(X)$ has a countable point-separating family. Therefore, $X$ continuously injects into $\mathbb{R}^{\omega}$.

To prove sufficiency we need the following claim.

Claim. $\mathbb{R}^{\omega}$ embeds into $C_{p}([0,1])$. 
To prove the claim, note that $C_{p}(\omega)=\mathbb{R}^{\omega}$ embeds into $C_{p}(\mathbb{R})$ since $\omega$ is closed in $\mathbb{R}$. By Gulko-Hmyleva theorem [4] that $\mathbb{R}$ and $[0,1]$ are $t$-equivalent, we conclude that, $\mathbb{R}^{\omega}$ embeds into $C_{p}([0,1])$. The claim is proved.

By Claim $X$ injects into $C_{p}([0,1])$. Let $F$ ne the image of such an injection. Due to homogeneity we may assume that the identity function is in $F$.Therefore, $F$ generates the topology of $[0,1]$. Consider the evaluation map the evaluation function $\Psi_{F}:[0,1] \rightarrow C_{p}(F)$. Since $F$ generates the topology of $[0,1]$, we conclude that $\Psi_{F}([0,1])$ genberate the topology of $F$. If $h: X \rightarrow F$ is a continuous bijection then the map $H: C_{p}(F) \rightarrow C_{p}(X)$ is a continuous injection, where $H(f)=h f$. Clearly, $H\left(\Psi_{F}([0,1])\right)=[0,1]$ is point separating.

Theorem 2.24. Let $X$ be a separable metric space. Then $C_{p}(X)$ has a topologygenerating subspace homeomorphic to $[0,1]$.

Proof. Embed $X$ into $C_{p}([0,1])$ so that the image $F$ contains the identity map. The evaluation function $\Psi_{F}:[0,1] \rightarrow C_{p}(F)$. Since $F$ generates the topology of $[0,1]$ and therefore $\Psi_{F}([0,1])$ generates the topology of $F$. Since $F=X$, we conclude that $[0,1]=\Psi_{F}([0,1])$ generates the topology of $F=X$.

Note that Theorem 2.24 cannot be reversed. Indeed, [0,1] generates the topology of $C_{p}([0,1])$ but the latter is not metrizable.

We would like to finish with two problems that are naturally prompted by our study.

Question 2.25. Characterize spaces $X$ for which $C_{p}(X)$ has a closed discrete point-separating subset.

Question 2.26. Characterize spaces $X$ for which $C_{p}(X)$ has a (closed) discrete topology-generating subset.

At last, the unattained goal of the paper is left as the following question.

Question 2.27. Assume that $C_{p}(X)$ has a discrete subspace of size iw $(X)$. Is it true that $C_{p}(X)$ has a discrete point-separating set? 


\section{REFERENCES}

[1] A. Arhangelskii, Topological Function Spaces, Math. Appl., vol. 78, Kluwer Academic Publishers, Dordrecht, 1992.

[2] R. Engelking, General Topology, PWN, Warszawa, 1977.

[3] V. V. Fedorchuk, A compact having a cardinality of continuum with no convergent sequences, Math. Proc. Cambridge Phil. Soc. 81(1977), 177-181

[4] S. Gulko and T. Hmyleva, Compactaness is not preserved by t-equivalence, Mat Zametki, vol 39, 6 (1986), 895-903.

[5] A. V. Ivanov, On bicompacta with hereditary separable finite powers, (in Russian) DAN SSSR, 243 (1978), 1109-1112.

[6] S. Mardesic, On covering dimension and inverse limits of compact spaces, Ill. J. of Math. 4 (1960), 278-291.

[7] P. Zenor, Hereditary m-separability and the hereditary m-Lindelöf property in product spaces and function spaces, Fund. Math. 106 (1980), 175-80.

E-mail address: Raushan_Buzyakova@yahoo.com

E-mail address: oleg@servidor.unam.mx

Facultad de Ciencias Fsico-Matematicas, Benemrita Universidad Autonoma de Puebla, Apdo postal 1152, Puebla, Puebla CP 72000, Mexico 